\title{
PREVALENCE OF THYROID DYSFUNCTION IN IRRITABLE BOWEL SYNDROME
}

Mohan Khadka ${ }^{1}$, Brindeshwori Kafle ${ }^{1}$, Sashi Sharma ${ }^{2}$, Prem Krishna Khadga ${ }^{2}$

\begin{abstract}
INTRODUCTION:

Irritable bowel syndrome (IBS) is a common public health problem. The condition is characterized by a scarcity of biological markers; thus, diagnostic definitions and classifications have relied to a large extent on symptoms, gastrointestinal manifestations of patients. While thyroid disorder should be considered in the differential diagnosis of patients with IBS symptoms, it is not clear if thyroid disturbances amongst patients with IBS are high enough to warrant routine screening. This study was done to see the prevalence of thyroid dysfunction in IBS patients and hence its significance
\end{abstract}

\section{MATERIALS AND METHODS:}

A cross-sectional study conducted at the Gastroenterology OPD of the Tribhuvan University Teaching Hospital, Kathmandu, Nepal between March 2015 and April 2016. All the consecutive patients presumed to be Irritable bowel syndrome by Rome III criteria were enrolled in the study. Thyroid Function Test (TFT) was performed in all patients in addition to routine investigations for the study group that include measurement of thyroid hormones (T3 and T4) together with thyrotropin stimulating hormone (TSH).

RESULTS:

Thyroid dysfunction particularly subclinical hypothyroidism was substantially found among patients with IBS

\section{CONCLUSION:}

Routine TFTs in patients with presumed IBS seems reasonable.

\section{KEYWORDS:}

Thyroid Disorders; Irritable bowel syndrome; Subclinical hypothyroidism

1. Asst. Professor, Department of Gastroenterology, Institute of Medicine, TU-TH, Kathmandu, Nepal

2. Professor, Department of Gastroenterology, Institute of Medicine, TU-TH, Kathmandu, Nepal

\author{
For Correspondence: \\ Dr. Mohan Khadka \\ Department of Gastroenterology, \\ Institute of Medicine, TU-TH, Nepal \\ Email:khamohan620@gmail.com
}




\section{INTRODUCTION}

Approximately 15 to $25 \%$ of the world population suffers from irritable bowel syndrome (IBS). ${ }^{1}$ IBS is a chronic condition characterized by abdominal discomfort or pain, abdominal bloating and changes in bowel habit. ${ }^{1,2}$ IBS can be classified according to the predominant bowel symptoms: IBS with constipation-predominant features (IBS-C), IBS with diarrhea-predominant features (IBS-D), IBS with mixed pattern of stool (IBS-M) and IBS with normal consistency stool (IBS-U). ${ }^{3}$ The condition is characterized by a scarcity of biological markers; thus, diagnostic definitions and classifications have relied to a large extent on symptoms, typically distinguished by persistent or recurrent abdominal pain related to defecation and/or chronic disturbance of bowel habits. ${ }^{3-5}$ The true incidence of gastrointestinal manifestations of patients with functional abnormalities of the thyroid is not well documented. Gastrointestinal motor dysfunction, manifested by altered intestinal motility and transit time, has widely been accepted as the leading cause of gastrointestinal symptoms of thyroid disease. ${ }^{6}$ Patients with hyperthyroidism can experience frequent bowel movements, diarrhea, even malabsorption with steatorrhea. ${ }^{6-8}$ Chronic dyspeptic symptoms such as epigastric pain and fullness, as well as eructation, nausea and vomiting are also frequently seen in these patients. Less commonly, hyperthyroidism has been reported to cause persistent and intractable vomiting. ${ }^{9}$ The overall decreased metabolic function seen in patients with hypothyroidism manifests in the GI tract with sluggish intestinal motility, ranging from mild constipation to paralytic ileus and colonic pseudoobstruction. ${ }^{10}$ While thyroid disorder should be considered in the differential diagnosis of patients with IBS symptoms, it is not clear if thyroid dysfunction amongst patients with IBS is high enough to warrant routine screening. Herein the findings from the prospective, crosssectional study has been analysed to see thyroid dysfunction in patients with IBS.

\section{MATERIALS AND METHODS}

This was a cross-sectional study conducted in the Gastroenterology OPD of the Tribhuvan University Teaching Hospital, Kathmandu, Nepal from May 2015 to February 2016. There was one study population who were consecutive adult patients with symptoms suggestive of IBS presented in the Gastroenterology OPD. Patients with suspected IBS fulfilled the Rome III criteria for IBS based on their responses to a questionnaire administered in the OPD. ${ }^{11}$ Patients were excluded from the study if they had been previously diagnosed with comorbid conditions that could have explained their GI symptoms (e.g. celiac disease, colon cancer, inflammatory bowel disease (IBD), scleroderma, small intestinal bacterial overgrowth, uncontrolled thyroid disease or diabetes). Patients with previous GI or intestinal (large or small bowel) surgery, with the exception of appendectomy or cholecystectomy, were also excluded. Patients reporting symptoms suggestive of organic diseases were excluded from the study and also women who were pregnant or breastfeeding or patients who had undergone previous diagnostic testing for their IBS symptoms were excluded from the study. No participants had been previously tested for thyroid function. Ethical clearance was taken as per the guidelines of the research Unit of Institute of Medicine.

\section{Collection of Blood Samples}

Venous blood sample (2-3 ml) was collected from antecubital vein in a plain vial, and was allowed to clot, and then subsequently serum was separated by centrifugation at $3000 \mathrm{~g}$ for 10 minutes and stored at $-20{ }^{\circ} \mathrm{C}$ until thyroid hormones were estimated.

\section{Assay procedure of thyroid hormones}

The thyroid hormones (fT3, fT4 and TSH) were assayed by Vitros ECIQ analyser. The reference range used for fT3 was $4.2-8.1 \mathrm{pmol} / \mathrm{L}$, for fT4 were $10-28.2 \mathrm{pmol} / \mathrm{L}$ and for TSH was 0.4-4.6 $\mathrm{mIU} / \mathrm{mL}$. Thyroid function was considered normal (Euthyroidism) when subjects had all the three hormones within the reference range. Abnormal thyroid function was further categorized as hyperthyroidism (increased fT3, fT4 and low TSH), subclinical hyperthyroid (both fT3 and fT4 are normal but low TSH), hypothyroidism (low fT3, fT4 and high TSH) and subclinical hypothyroidism (fT3, fT4 are normal but high TSH level) (Peter, 2009; Helfand and Redfern, 1998).

\section{Principle of the Assay}

The quantitative immunoenzymatic determination of TFT (TSH, T3 \& T4) is based on the ELISA (Enzyme-linked Immunosorbent Assay) technique. The assay system utilizes two monoclonal antibodies specific for different antigenic determinants of each of the (TSH, T3 \& T4). The first antibody is immobilized on the surface of the microtiter wells. The second antibody is conjugated to horseradish peroxidase. The test sample is allowed to react simultaneously with the two antibodies, resulting in the (TSH, T3 \& T4) molecules being sandwiched between the solid phase and the enzyme linked antibodies. After an incubation step, the wells are washed with Washing Solution to remove all unbound material. The immune complex is visualized by adding Tetramethylbenzidine (TMB) substrate which gives a blue reaction product. The intensity of this product is proportional to the concentration of (TSH, T3 \& T4) in the specimen. Sulphuric acid is added to stop the reaction. This produces a yellow 
endpoint colour. Absorbance is read using an ELISA microwell plate reader. The color intensity is directly proportional to the concentration of (TSH, T3 \& T4) present in the test sample (instruction manual for autobio diagnostics Thyroid ELISA kits).

\section{Statistical Analysis}

Statistical analysis was done using Excel system version 2007 and includes descriptive statistics (mean and standard deviation) and inferential statistics (Chi-square test) to test the significance of difference. When P value is less than 0.05 , the difference is considered significant, and the difference is considered highly significant when $\mathrm{P}$-value was less than 0.001 .

\section{RESULTS}

A total of 80 patients with symptoms suggestive of IBS were enrolled in the study from May 2015 to February 2016. There were $42(52.5 \%)$ females and $38(47.5 \%)$ males with age range of 62 with minimum of 18 and maximum of 80 years. The mean age was 34 years. Majority of the subjects were female. Among them 47 were Irritable Bowel Syndrome Diarrhea predominant (IBS-D), 18 were Irritable Bowel Syndrome Constipation predominant (IBS-C), 8 were Irritable Bowel Syndrome unsubtype (IBS-U) and 7 were Irritable Bowel Syndrome Mixed (IBS-M).

In this study, subjects are divided according to the thyroid status which shows subclinical hypothyroidism 12 (15\%), overt hypothyroidism $2(2.5 \%)$, overt hyperthyroidism 1 $(1.25 \%)$ and euthyroidism $65(81 \%)$ as shown in Fig 1 pie chart.

Overall prevalence of thyroid disorder was found to be $19 \%$ with subclinical hypothyroidism being the most common $15.0 \%$. Table 1 shows the overall statistics of thyroid hormones. Fig 2 shows the prevalence of thyroid dysfunction among different subgroups of IBS. The prevalence of thyroid dysfunction was $19 \%$ in IBS-D, $27.8 \%$ in IBS-C, $14 \%$ in IBS$\mathrm{M}$ and no dysfunction in IBS-U. There was no significant difference in prevalence of thyroid dysfunction among different subgroups of IBS (Pearson Chi-square $=2.906$, Pvalue $=.406)$. Fig 3 shows the prevalence of different types of thyroid dysfunction among different IBS subtypes. Out of 47 IBS-D patients, subclinical hypothyroidism was present in 8 and overt hypothyroidism in 1 whereas 38 were euthyroid. Out of 18 IBS-C patients, subclinical and overt hypothyroidisms were present in 4 and 1 respectively whereas 13 were euthyroid. There was 1 overt hyperthyroidism out of 7 patients with IBS-M whereas 6 were euthyroid. All 8 patients of IBS-U were euthyroid.
Fig 1

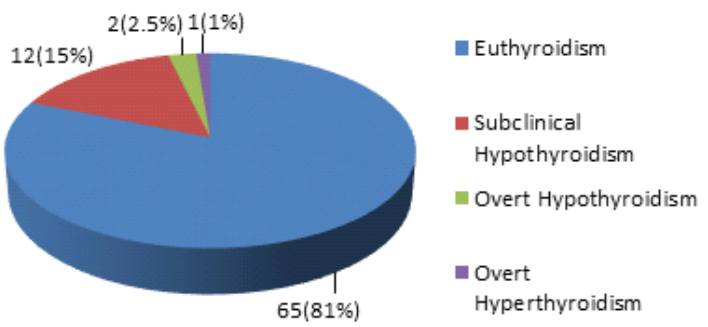

Table 1

\begin{tabular}{|l|c|c|c|}
\hline \multicolumn{4}{|c|}{ Statististics of Thyroid hormones } \\
\hline & T3 & T4 & TSH \\
\hline Total & 80 & 80 & 80 \\
\hline Mean & & & \\
\hline Median & 6.05 & 14.73 & 2.85 \\
\hline Std. Deviation & 6.10 & 14.20 & 2.20 \\
\hline Range & 1.02 & 3.64 & 2.18 \\
\hline Minimum & 4.10 & 18.60 & 11.80 \\
\hline Maximum & 8.20 & 9.00 & .20 \\
\hline
\end{tabular}

Fig 2

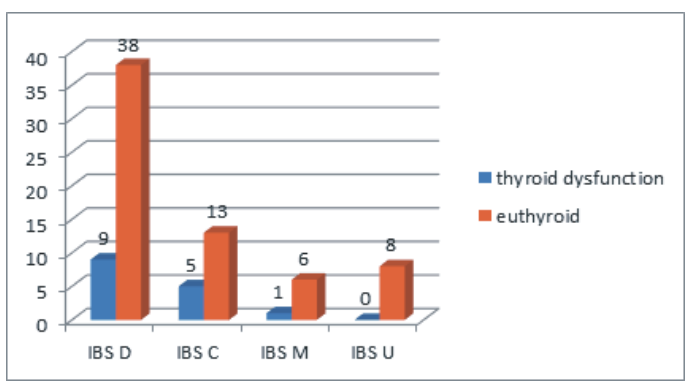

Fig 3

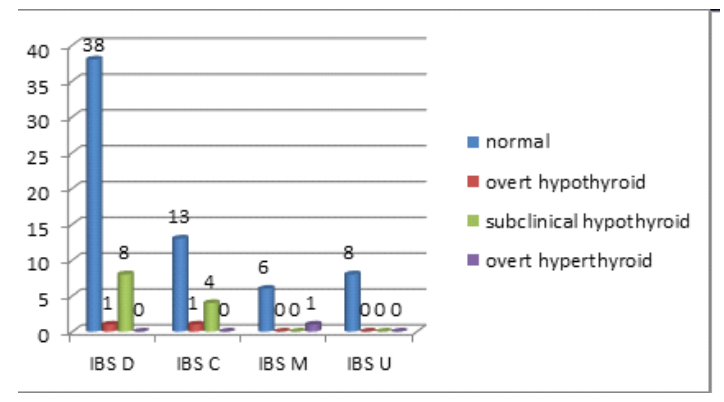

\section{DISCUSSION}

In the present study more than half of the subjects are females 
indicating that more females are suffering from thyroid illness. This finding is supported by few studies. ${ }^{12,13}$

The National Health and Nutrition Examination Survey (NHANES 1999-2002) of 4392 individuals reflecting the US population reported hypothyroidism in $3.7 \%$ of the general population. ${ }^{14}$ In a population-based study done in Cochin, India on 971 adult subjects, the prevalence of hypothyroidism was $3.9 \%$ and subclinical hypothyroidism was $9.4 \%{ }^{15}$

The prevalence of thyroid disorders seen in a study conducted in high risk groups at tertiary center in IOM, TUTH, Nepal was $29.0 \% .^{16}$ Similar study observed $30 \%$ of the population suffering from thyroid dysfunction in high risk groups at a tertiary referral center in eastern Nepal. ${ }^{17}$ The worldwide prevalence of hypothyroidism in various studies shows a remarkable variation and current prevalence ranges from $1 \%$ to $20 \%$ for sub-clinical and $1-2 \%$ for overt hypothyroidism. ${ }^{18}$

In our study, the prevalence of thyroid dysfunction in IBS i.e. $19 \%$ was higher than in general population that were conducted in USA ${ }^{14}$ and India. ${ }^{15}$ However it was comparable to the study conducted in high risk groups ${ }^{16,17}$ In the present study since the prevalence of thyroid dysfunction in IBS was high which clearly indicates that IBS is a high risk group for the possible association of thyroid dysfunction.

According to the results of the current study, altered thyroid function was found in patients with suspected IBS. These findings were in disagreement with the findings of Hamm et. at ${ }^{19}$ who failed to modify the criteria for diagnosis of IBS. The mechanism behind our results was not clear; however, direct hormonal effects or stimulatory actions in the central nervous system have been suggested. Treatment of the thyrotoxicosis with beta blocking agents and anti-thyroid drugs greatly improves these symptoms. ${ }^{20}$ The exact biochemical mechanisms by which thyroid disorders affect the gastrointestinal system are not fully understood. Changes may be seen on a molecular level with alteration of hormone receptors, dysfunction of the autonomic nervous system and myoelectrical enteric activity, as well as changes on a tissue level in the form of myopathy. ${ }^{21}$ Hormonal effects on the GI tract may be a direct result of thyroid hormone, or as a result of synergistic effects of catecholamines. Tenore et al. investigated the effect of thyroxin (T4) on the intestinal chloride/bicarbonate exchange in hypo- and hyperthyroid rats. They found that alterations in intestinal ion exchange, mainly the flux of chloride, led to mucosal effects and resultant diarrhea. These findings were not seen when T4 was added ex vivo to rat ileum, suggesting that the effect on electrolyte transport likely requires systemic factors. ${ }^{22}$ Furthermore, the beta-adrenergic antagonist propranolol inhibits intestinal transit in hyperthyroidism, thereby indicating that some of the dysmotility may be mediated through the adrenergic/catecholamine system. ${ }^{23}$ Dysfunction of the autonomic nervous system (ANS) may modify the neuro-hormonal milieu, and result in alterations of myoelectric activity. However, there are no clear data regarding the exact mechanisms involved. Hypothyroidism characteristically results in accumulation of glycosaminogly cans, mostly hyaluronic acid, in interstitial tissues throughout the body. This leads to interstitial edema that is particularly evident in the skin, heart muscle, and skeletal muscle, but also in the gastric smooth muscle. Edema in the gastric muscle may predispose to abnormalities of gastric myoelectric activity and thus the dysmotility seen in hypothyroid patients. ${ }^{24}$ Examination of TFTs in patients with an established history of IBS revealed an altered function of thyroid comparable to that in the general population; this might be attributed to high incidence of thyroid problems in older adults. ${ }^{25}$

\section{CONCLUSION}

The prevalence of thyroid dysfunction in IBS patients is high. Therefore, the routine thyroid function tests in the diagnostic evaluation of established IBS patients should be recommended.

\section{REFERENCES}

1. Thompson WG: A world view of IBS. In: Irritable Bowel Syndrome: Diagnosis and Treatment. 1st edition. Camilleri $M$ and Spiller R (eds). Saunders, New York, NY, pp 17-26, 2002.

2. Everhart JE and Renault PF: Irritable bowel syndrome in officebased practice in the United States. Gastroenterology 100: 9981005, 1991.

3. Thompson $W G$, Dotevall G, Drossman DA, Heaton KW, Kruis $W$. Irritable bowel syndrome: guidelines for the diagnosis. Gastroenterol Int. 1989;2:92-95.

4. Drossman DA, Thompson WG, Talley NJ, Funch-Jensen P, Janssen J, Whitehead WE. Identifications of subgroups of functional gastrointestinal disorders. Gastroenterol Int. 1990;3:159-72.

5. Wegener M, Wedmann B, Langhoff T, Schaffstein J, Adamek R. Effect of hyperthyroidism on the transit of a caloric solid li quid meal through the stomach, the small intestine, and the colon in man. JClin Endocrinol Metab 1992; 75: 745749.

6 Tinker MB. Discussion of paper by Verbrycke JR. Masked gastrointestinal hyperthyroidism. JAMA 1931; 97: 515-516.

7. Karaus M, Wienbeck M, Grussendorf M, Erckenbrecht JF, Strohmeyer G. Intestinal motor activity in experimental hyperthyroidism in conscious dogs. Gastroenterology 1989; 97: 911-919. 
8. Hoogendoorn EH, Cools BM. Hyperthyroidism as a cause of persistent vomiting. Neth J Med 2004; 62: 293-296.

9. Bassotti G, Pagliacci MC, Nicoletti I, Pelli MA, Morelli A. Intestinal pseudoobstruction secondary to hypothyroidism. Importance of small bowel manometry. J Clin Gastroenterol. 1992; 14: 56-58.

10. Cash BD, Schoenfeld PS, Chey WD. The utility of diagnostic tests in irritable bowel syndrome patients: a systematic review. Am J Gastroenterol. 2002; 97:28129.

11. Friedman MN (1999) Screening for thyroid disease. Ann. Med. 130: $161-162$.

12. Ladenson Paul $W$ (2000). American thyroid association guidelines for detection of thyroid dysfunction. Arch Intern Med. 160: 1573-1575. DOI: 10.1001/archinte.160.11.1573.

13. Aoki Y, Belin RM, Clickner R, et al. Serum TSH and total T4 in the United States population and their association with participant characteristics: National Health and Nutrition Examination Survey (NHANES 1999-2002). Thyroid. 2007 Dec. $17(12): 1211-23$.

14. Usha Menon V, Sundaram KR, Unnikrishnan AG, Jayakumar $R V$, Nair V, Kumar H. High prevalence of undetected thyroid disorders in an iodine sufficient adult south Indian population. $J$ Indian Med Assoc 2009; 107:72-7.

15. R.V. Mahato et al. (2015) Int J Appl Sci Biotechnol, Vol 3(1). 119-122.

16. Baral N, Lamsal M and Koner BC (2002). Thyroid dysfunction in eastern Nepal. South Asian J. Trop. Med. Public Health 33: 638-641.
17. Aminorroaya A, Janghorbani $M$, Amini $M$, Hovsepian $S$ Tabatabaei A and Fallah Z (2009) The prevalence of thyroid dysfunction in an iodine-sufficient area in Iran. Arch. Iran Med. 12(3): 262-270.

18. Hamm LR, Sorrells SC, Harding JP, Northcutt AR, Heath AT, Kapke GF, Hunt CM, Mangel AW. Additional investigations fail to alter the diagnosis of irritable bowel syndrome in subjects fulfilling the Rome criteria. Am J Gastroenterol. 1999 May; 94(5): 1279-82.

19. Hoogendoorn EH, Cools BM. Hyperthyroidism as a cause of persistent vomiting. Neth JMed 2004; 62: 293-296.

20. Karaus M, Wienbeck M, Grussendorf M, Erckenbrecht JF, Strohmeyer G. Intestinal motor activity in experimental hyperthyroidism in conscious dogs. Gastroenterology 1989; 97. 911-919.

21. Tenore A, Fasano A, Gasparini N, Sandomenico ML, Ferrara A, Di Carlo A, Guandalini S. Thyroxine effect on intestinal Cl/HCO3- exchange in hypo- and hyperthyroid rats. J Endocrinol 1996; 151: 431-437.

22. Thomas FB, Caldwell JH, Greenberger NJ. Steatorrhea in thyrotoxicosis. Relation to hypermotility and excessive dietary fat. Ann Intern Med 1973; 78: 669-675.

23. Greenspan FS, Rapaport B. Thyroid gland. In Basic and Clinical Endocrinology FS Greenspan, JD Baxter (eds). Norwalk, Connecticut, Appleton and Lange, 1992: 188246.

24. Jalal NA, Al-Samarrai AH, Al-Tikriti KA. Biochemical changes in patients with hyperthyroidism. Tikrit Journal of Pure Science Vol. 15 No.(1) 2010. 